\title{
Laryngeal Nerve Activity During Pulse Emission in the CF-FM Bat, Rhinolophus ferrumequinum
}

\author{
II. The Recurrent Laryngeal Nerve \\ Rudolf Rübsamen and Gerd Schuller* \\ Arbeitskreis Neuro- und Rezeptorphysiologie, Fachbereich Biologie der J.W. Goethe-Universität, D-6000 Frankfurt/Main, \\ Federal Republic of Germany
}

Accepted March 5, 1981

Summary. The activity of the recurrent laryngeal nerve (RLN) was recorded in the greater horseshoe bat, Rhinolophus ferrumequinum. Respiration, vocalization and nerve discharges were monitored while vocalizations were elicted by stimulation of the central gray matter. This stimulation evoked either expiration or expiration plus vocalization depending on the stimulus strength. When vocalization occurred it always took place during expiration.

Recordings from the RLN during respiration showed activity during the inspiration phase, but when vocalization occurred there was activity during inspiration and expiration. These results are consistent with the view that the RLN innervates muscles which control the opening and closing of the glottis. During vocalization the vocal folds are closely approximated and the discharge patterns of the nerve suggests that it controls the muscles which start and end each pulse.

\section{Introduction}

Bats produce orientation calls with the larynx and extract information about prey and other aspects of their environment from the reflected echoes. The accuracy of echolocation depends both on the resolution of the auditory system and the precision of sound production. Each orientation call of Rhinolophus ferrumequinum consists of a long $(10-150 \mathrm{~ms})$ constant frequency (CF) portion of about $83 \mathrm{kHz}$ and a final frequency modulated (FM) part during which the fre-

* Supported by grants of the Deutsche Forschungsgemeinschaft (DFG), Br 593/2, Schu 390/1, 2, Ne 146/8, and SFB 45

Abbreviations: $R L N$ Recurrent laryngeal nerve; $C F$ Constant frequency; $F M$ Frequency modulation; $S L N$ Superior laryngeal nerve; $A S T$ Ante stimulus time quency has a downward $15-20 \mathrm{kHz}$ sweep within 3$5 \mathrm{~ms}$ (Schnitzler 1968). During flight the frequency of the emitted sounds is lowered in order to compensate for Doppler shifts due to he relative velocity between the bat and obstacles (Schnitzler 1968; Schuller et al. 1974). When approaching a target the durations of the orientation calls are shortened and the repetition rate of the sounds is increased up to $80 / \mathrm{s}$.

The frequency patterns, temporal sequences, durations and intensities of the emitted sounds are controlled by the larynx. The superior laryngeal nerve (SLN) innervating the $\mathrm{m}$. cricothyroideus, controls the frequency of the emitted calls (Novick and Griffin 1961; Suthers and Fattu 1973; Schuller and Suga 1976). Schuller and Rübsamen (see companion paper) recorded nerve activity from the SLN as a function of the emitted frequency and found a linear relationship between the discharge rate of the nerve and the frequency of the orientation call; this linearity matched the discharge rate to frequency relationship also found for the $\mathrm{m}$. cricothyroideus (Schuller and Suga 1976).

While the SLN innervates the $\mathrm{m}$. cricothyroideus, the recurrent larnygeal nerve (RLN) controls the other intrinsic laryngeal muscles (Bowden and Scheuer 1961). Most of the muscles innervated by the RLN are attached to the arytenoid cartilage and influence the position and tension of the vocal folds (Negus 1949). The RLN innervates antagonistic muscle systems, e.g. the $\mathrm{m}$. cricoarytenoideus posterior, which widens the vocal folds and the $\mathrm{m}$. cricoarytenoideus lateralis, which closes the larynx. Therefore, cutting the recurrent laryngeal nerve is not a useful technique for functional analysis of the intrinsic muscles (Novick and Griffin 1961; Suthers and Fattu 1973; Schuller and Suga 1976). In vespertilionid bats bilateral RLN-cutting does not markedly change the structure of emitted sonar signals (Novick and Griffin 
1961; Suthers and Fattu 1973). When the RLN is bilaterally cut in Rhinolophus ferrumequinum the bats suffocate unless a tracheotomy is carried out before hand (Schuller and Suga 1976).

In Rhinolophus ferrumequinum the vocal folds, and perhaps the vestibular folds as well, seem to function like a valve controlled by the recurrent laryngeal nerve, and the RLN may therefore be responsible for the control of the onset and the termination of an echolocation pulse. This paper describes the RLN activity correlated with the respiratory cycle and the emission of echolocation pulses in the horseshoe bat.

\section{Materials and Methods}

Recordings of the nerve activity were made in five greater horseshoe bats, Rhinolophus ferrumequinum. Before each experiment the duration and frequency content of the orientation pulses of the animal were measured. Under Nembutal ${ }^{\circledR}$ the skull of the animal was exposed and a short rod was mounted on the bone with dental acrylic (Paladur). The animal was placed in a holder and the head was fixed by placing the rod in a rigid clamp.

A tungsten electrode was inserted into the central grey matter for electrically eliciting echolocation pulses (for details see Suga et al. 1973). The electrical stimuli consisted of $15 \mathrm{~ms}$ bursts of 15 negative square waves with $0.1 \mathrm{~ms}$ durations. The orientation pulses of the bat were picked up with a Brüel \& Kjaer microphone (Type 4136), amplified (Hewlett \& Packard, Model 465A Amplifier) and fed to a frequency/voltage converter (custom made with a frequency/voltage integrated circuit, IC9400) which provided frequency information on the echolocation pulses. The envelopes of the pulses were obtained from a double wave rectifier (custom made) yielding information on the intensity and time course of the echolocation signal.

The respiratory activity of the animal was monitored with a hot wire anemometer located $2 \mathrm{~mm}$ in front of the nostrils. The anemometer consisted of a small $(10 \mu \mathrm{m}$ diameter $)$ platinumiridium wire connected to a bridge circuit and differential amplifier. The dynamic response of the anemometer system was tested with a low-frequency loudspeaker and yielded a time constant of $7 \mathrm{~ms}$.

For laryngeal surgery the animal was placed in a supine position. The ventral part of the neck was opened caudal to the larynx and both recurrent laryngeal nerves, which parallel the trachea, were exposed. During recordings the wound margins were continuously treated with a local anaesthetic (Novocain ${ }^{\circledR}$ ). Whole nerve recordings were done with a small tungsten wire $(50 \mu \mathrm{m}$ diameter, sharpened by electrolysis and bent into a small hook) which was fixed to an elastic spiral spring made of soft, $100 \mu \mathrm{m}$ thick silver wire. The tip of the hook was fixed in the RLN and the spring suspension prevented the hook from slipping out of the RLN when the larynx moved during respiration and vocalization. The indifferent electrode was fixed in the neck muscles. This arrangement enabled multifiber-recordings without violating the larynx.

The activity of the RLN, the animal's vocalizations, the outputs of the frequency/voltage converter, the pulse envelope monitor and the anemometer were recorded on a four channel analogue tape recorder (Philips Analog 7) for later processing. The recorded nerve activity was fed to a window comparator for histogram building. The threshold for the window comparator was set by auditory control to separate the activity correlated to respiration and vocalization from the spontaneous background activity.

Hans Zöller provided a program for our computer system (Digital equipment PDP 11/40) that constructed histograms of the nerve activity. The trigger point corresponded to a certain point in the respiration cycle or to the end or the beginning of an echolocation pulse. The program also processed RLN-activity prior to the triggering stimulus and therefore provided Ante-StimulusTime-Histogram (AST-histogram).

\section{Results}

\section{Recordings of Respiration}

The respiratory rhythm in Rhinolophus ferrumequinum varied between 3 and $5 \mathrm{~Hz}$ depending on the individual. Each expiration was a short pulse of air expelled through the nostrils. Expiration lasted about $50 \mathrm{~ms}$ (Fig. 1, sharp downward peak). The inspiration cycle was slower than the expiration cycle and lasted about 100-150 ms (Fig. 1, shallow downward peak). The following positive wave in the anemometer output is caused by compensatory effects of the electronic equipment. When the bat vocalized spontaneously the rhythm of respiration did not change and the echolocation pulses were strictly synchronized to the expira-

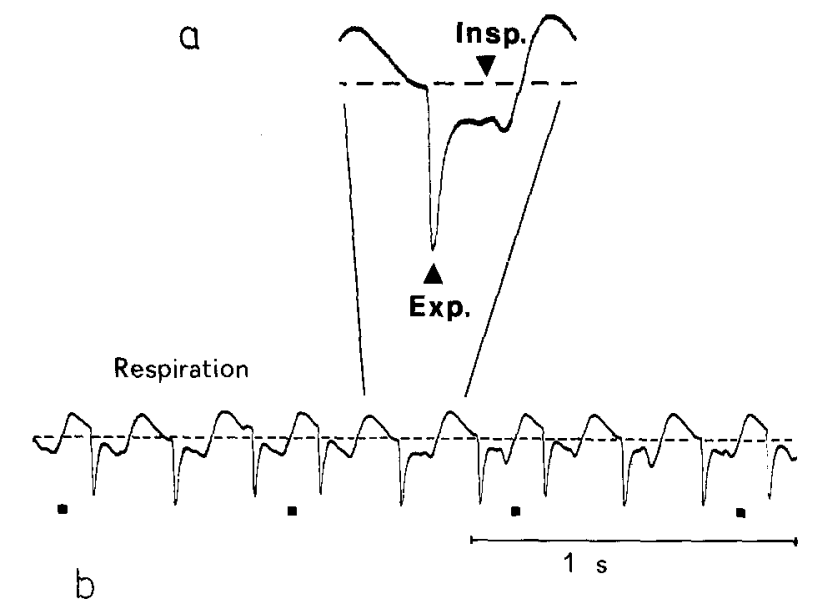

Respiration
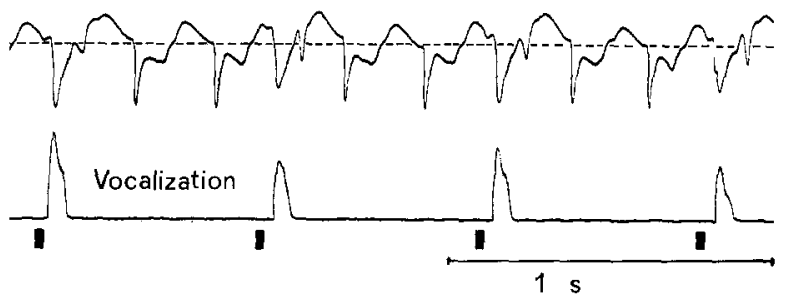

Fig. 1a, b. Examples of synchronization of respiration and vocalization after electrical stimulation of the central gray matter. a Upper trace: The output of the anemometer showing expiration (sharp downward peaks) and inspiration (shallow downward peaks). The slow positive wave above the dashed line is caused by compensatory effects of the electronic equipment. Lower trace (black squares): The low amplitude electrical stimulation $(10 \mu \mathrm{A})$ which locks expiration to the stimulus with a latency of $82 \mathrm{~ms}$ (Insp. Inspiration, Exp. Expiration). b High amplitude $(15-20 \mu \mathrm{A})$ electrical stimulation (black rectangles) which simultaneously triggers deeper expiration (upper trace) and vocalization of an echolocation call (lower trace). Latency is $44 \mathrm{~ms}$ 


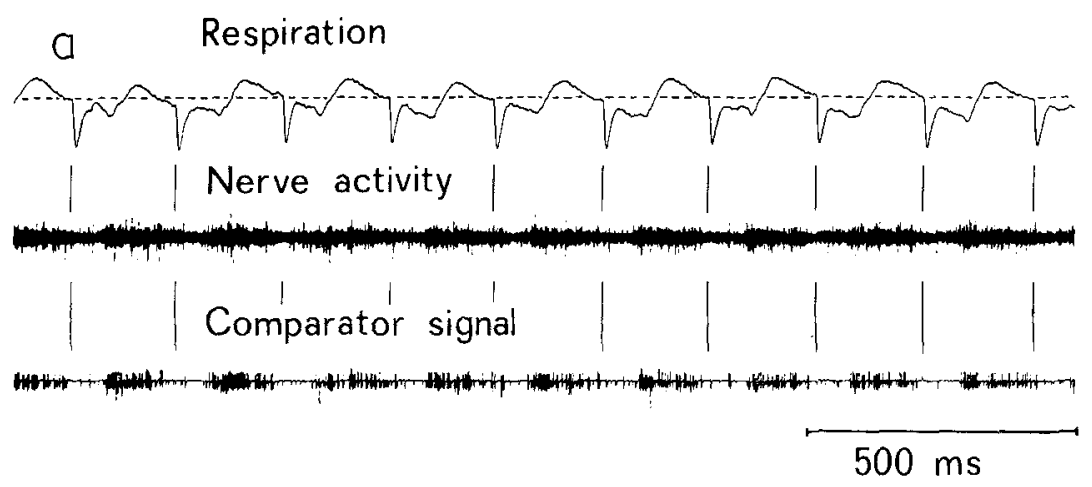

b

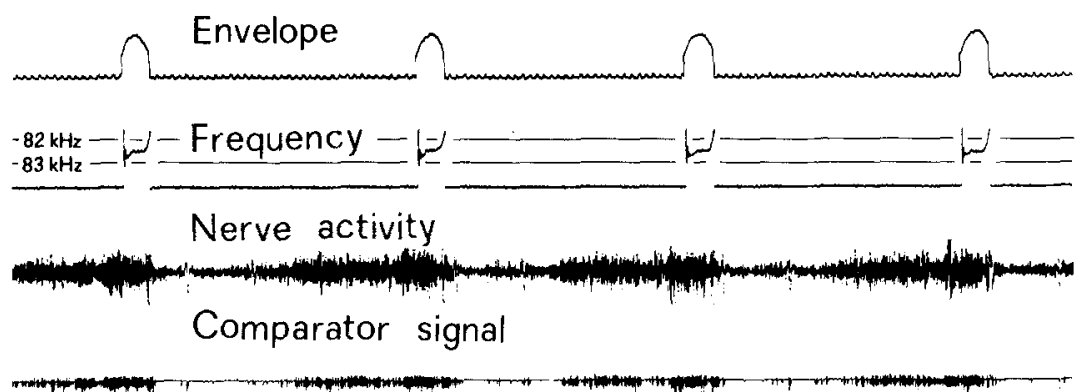

$500 \mathrm{~ms}$
Fig. 2a, b. Recording of fiber activity of the recurrent laryngeal nerve. a Registration of RLN activity in association with respiration. In the upper trace respiration is indicated by the output of an anemometer. Middle trace: nerve activity; lower trace: comparator signal of the nerve recording used for histogram-building in Fig. 3 a b Recording of RLN activity in association with respiration and vocalization. Upper trace: envelope of the orientation calls. Second trace: frequency signal of these calls. Third trace: gross activity of the nerve. Fourth trace comparator signal used for histogram formation in Fig. 3b tion cycle. Figure $1 \mathrm{~b}$ shows a series of bat calls which were elicited by synchronized electrical stimulation to every fourth respiration cycle. During the vocalization the expiratory pulse was deeper and longer than without vocalization, i.e., more air was pressed through the larynx during vocalization. The volume of inhalation was also larger after vocalization.

Electrical stimulation in the central gray matter affected respiration and elicited vocalization. With low stimulus intensities (stimulus burst $=15$ stimuli of $0.1 \mathrm{~ms}$ each, burst duration $15 \mathrm{~ms}$, peak stimulus current $10 \mu \mathrm{A}$ ) synchronized expiratory pulses occurred with a latency of about $80 \mathrm{~ms}$ (Fig. 1a). In between the stimulus bursts the respiration continued with its spontaneous rhythm. If the stimulus intensity was increased to $15-20 \mu \mathrm{A}$ the latency of the expiration pulse decreased to about $45 \mathrm{~ms}$ and concurrently a vocalization signal was elicited (Fig. 1 b). As demonstrated, the electrical stimulation overrode the spontaneous rhythm of respiration and synchronized the respiration and echolocation pulse emission to the artificial stimulus. If stimulus intensity was further increased the expiration cycle lengthened and multiple pulse emission occurred during the prolonged expiration, but no stable recordings were possible since the animal also started to move and became excited.

Respiration was affected in its rhythm immediately after unilateral sections of the RLN but it returned to normal within a few minutes. The effect of unilater- al RLN-sections on vocalization was observed in one animal over a period of 14 days; the frequency of the CF-portion of the echolocation sound dropped by 1 to $2 \mathrm{kHz}$, whereas the average duration of the echolocation pulse was not affected.

\section{Recording of RLN-Activity During Respiration and Vocalization}

During quiet respiration, without concurrent vocalization, the RLN showed activity that was correlated with the different parts of the respiration cycle. The nerve fibers strongly discharged during the inhalation period (Fig. 2a), whereas the activity was reduced as long as expiration occurred. In Fig. 3 a the RLN activity is summed over 30 cycles of respiration in an AST-histogram triggered by the beginning of the expiration period. The strong nerve discharges during inhalation lasted from $150 \mathrm{~ms}$ to $25 \mathrm{~ms}$ before the onset of expiration; the activity during expiration was reduced to the spontaneous level.

When the bat was vocalizing the activity during inhalation remained unaffected, but a pronounced peak of nerve activity appeared $20 \mathrm{~ms}$ prior to the onset of vocalization, i.e. expiration (Fig. 2b). During the CF-portion of the sound the nerve maintained a certain level of activity that sharply dropped at the end of the CF-portion. The terminal FM-sweep of the echolocation sound was preceded by a short, 

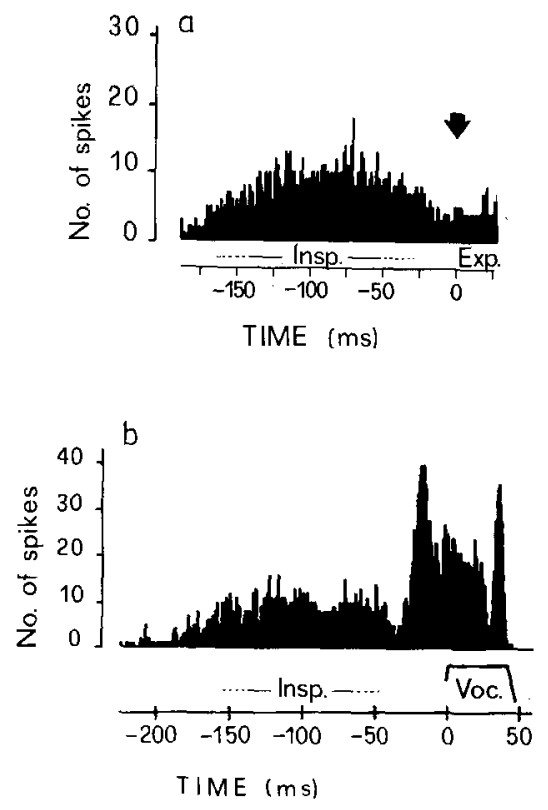

Fig. 3a, b. Ante-Stimulus-Time-Histogram of the RLN activity during respiration and vocalization. a Activity of the nerve during respiration without concurrent vocalization. The histogram is triggered by the start of the expiration phase (arrow). The histogram is composed of an average of 30 different cycles (Insp. Inspiration; Exp. Exspiration). b Nerve activity during vocalization. This histogram is triggered by the onset of vocalization. The position of the orientation calls is presented by a sketch of the frequency pattern of the orientation call. The histogram is composed of an average of 30 different cycles (Insp. Inspiration; Voc. Vocalization)

narrow burst of strong RLN-activity. The AST-histograms of the nerve activity during the emission of echolocation pulses shows the fine structure of the discharge pattern with the activity burst prior to the beginning and end of the call (Fig. $3 \mathrm{~b}$ ). The starting point for histogram construction was triggered by the onset of vocalization. This discharge pattern during vocalization was found in all RLN recordings and no difference between the left or right recurrent laryngeal nerves was observed.

\section{Discussion}

The larynx plays an important role in the control of air flow in association with vocalization and respiration. There has been much interest in laryngeal mechanisms in bats where a high degree of coordinated activity must exist. The pattern of respiration has been measured in several species of bats (Schnitzler 1968; Suthers et al. 1972; Roberts 1972). These authors found roughly sinusoidal respiratory cycles without the pronounced expiration pulse found in this study (Fig. 1). This discrepancy in waveform is due to different monitoring methods for respiration; Schnitzler (1968) used body movements of the animal;
Suthers et al. (1972) and Roberts (1972) used hot wire anemometers with low dynamic responses. In all of these experiments it has been shown that there is a clear coincidence of vocalization with the expiratory phase of respiration. Due to the fast response of the anemometer used in our experiments the fine structure of the respiratory cycle, characterized by a pronounced expiratory pulse, could be demonstrated. The duration of the expiratory pulse of about $50 \mathrm{~ms}$ coincides with the average duration of the echolocation pulses in Rhinolophus ferrumequinum. A close relationship between echolocation pulse emission and expiration phase of the respiratory cycle was also revealed. Here it was clear that low intensity stimuli synchronized the expiration phase to the stimulus and high intensity stimuli additionally elicited vocalization. The consistent occurrence of vocalization during the respiratory phase proposed for Rhinolophus ferrumequinum by Schnitzler (1968) and Roberts (1972) can therefore be confirmed.

Recordings from the RLN in bats showed that during quiet respiration the inspiratory cycle is accompanied by a strong discharge of recurrent laryngeal nerve fibers. This finding coincides with the experimental results of Hast (1967), Suzuki and Kirchner (1969), Suzuki et al. (1970) and Horiuchi and Sasaki (1978) who worked on other types of mammals. They showed that the $\mathrm{m}$. cricoarytenoideus posterior is active during inhalation but not during expiration. The recorded activity may therefore arrise from RLN fibers innervating these muscles which widens the aperture between the vocal folds.

During vocalization two distinct, sharp activity bursts were recorded in the RLN of Rhinolophus ferrumequinum, one prior to the onset of vocalization and one just prior to the final FM portion of the echolocation sound (Fig. 2b). These activities may be related to RLN fibers that innervate the intrinsic laryngeal muscles which narrow or close the vocal folds. The activation of these muscles before the onset of vocalization and thus prior to the expiratory phase should lead to a build up of the subglottic pressure when the expiratory cycle begins. Vocalization is synchronized to a sharp increase in subglottic pressure and starts at the moment when the subglottic pressure reaches its plateau (Schuller, unpublished results). The subglottic pressure is maintained throughout the emission of the echolocation pulse and breaks down at the end of vocalization. The RLN activity just prior to the final FM portion of each pulse may be attributed to fibers that cause an active opening of the vocal folds so that the subglottic pressure breaks down. The RLN activity at the termination of the echolocation pulse glottic may therefore be responsible mainly for the reduction of the subglottic 
pressure and consequently for the amplitude decay to zero at the termination of the pulse. We have no evidence that the RLN activity is associated with the tuning properties during the production of the FM sweep. The role of the internal laryngeal muscles in sound emission in bats may be compared to that of a valve which regulates the subglottic pressure. The $\mathrm{m}$. cricoarytenoideus lateralis leads to the closure of the valve and the $\mathrm{m}$. cricoarytenoideus posterior opens the valve. Elias (1908) and Beissmann (1975) showed that in Rhinolophus ferrumequinum these two sets of muscles are especially well developed while the rest of the intrinsic laryngeal muscles are relatively small. These morphological data support the mechanisms suggested above for echolocation sound emission in Rhinolophus ferrumequinum.

Under the assumption that the RLN in Rhinolophus ferrumequinum follows the innervation pattern as in other mammals and performs similar functions, the control function of the RLN in sound production may be summarized as follows: During the inspiration phase the larynx (glottis) is opened by contraction of the posterior cricoarytenoid muscles. Then prior to the onset of vocalization the larynx is closed by the activity of the $\mathrm{m}$. cricoarytenoideus lateralis, showing up in a pronounced activity of the RLN $50 \mathrm{~ms}$ prior to the onset of vocalization. Contraction of the abdominal muscles builds up a high subglottic pressure. Since the beginning of vocalization is not marked by a pronounced RLN discharge, the vocal folds might be opened passively and the air forced to flow between them. The tension of the vocal cords is controlled by the activity of the cricothyroid muscle (Schuller and Suga 1976; Schuller and Rübsamen, companion paper) and perhaps to some extent by the thyroarytenoid muscles, since after unilateral RLN-section the frequency of the CF-part of the orientation call decreases by 1 or $2 \mathrm{kHz}$. At the end of vocalization different events coincide: the frequency of the sound decreases for a total of 15$20 \mathrm{kHz}$ and the subglottic pressure diminishes. This means that the antagonistic system is activated and the vocal folds are widened. The widening may be affected by the activity of the posterior cricoarytenoid muscle which rotates the arytenoid cartilagues and moves the vocal cords to a lateral position. The simultaneous stop of the expiratory pulse indicates that there is fine coordinated control of abdominal and laryngeal muscles.

Gacek (1975) showed with injections of horseradish peroxidase that in cats the laryngeal muscles innervated by the SLN and RLN receive motorneuron input from two brainstem nuclei, the nucleus ambiguus and the nucleus retrofacialis. He pointed out that this double innervation might be the morpho- logical substrate for the control of the larynx during expiration and inspiration. Pitts (1949) however showed a dorsolateral organization of the expiratory and inspiratory motorneurons in the brainstem. Thus the double innervation of the larynx reflects on the fact that the larynx is involved in both respiration and vocalization.

We are indebted to H. Zöller for providing the computer programs and to $O . W$. Henson for discussion and critical reading of the manuscript.

\section{References}

Beissmann E (1975) Morphologische Untersuchungen am Kehlkopf der echoortenden Fledermaus Rhinolophus ferrumequinum. Staatsexamensarbeit, Frankfurt/M

Bowden REM, Scheuer IL (1961) Comparative study of the nerve supply of the larynx in Eutherian mammals. Proc Zool Soc (Lond) 136:325-330

Elias H (1908) Zur Anatomie des Kehlkopfes der Mikrochiropteren. Morphol Jahrb 37:70-119

Gacek RR (1975) Localization of laryngeal motor neurons in the kitten. Laryngoscope 85:1841-1861

Hast HM (1967) The respiratory muscle of the larynx. Ann Otol Rhinol Laryngol 76:489-497

Horiuchi M, Sasaki CT (1978) Cricothyroid muscle in respiration Ann Otol Rhinol Laryngol 87:386-391

Negus VE (1949) The comparative anatomy of the larynx. Grune and Stratton, New York

Novick A, Griffin DR (1961) Laryngeal mechanisms in bats for the production of the orientation sounds. J Exp Zool 148:125145

Pitts RF (1949) Organization of the neural mechanisms responsible for rhythmic respiration. In: Fulton JF (ed) Textbook of physiology. Saunders, Philadelphia

Roberts LH (1972) Correlation of respiration and ultrasound production in rodents and bats. J Zool (Lond) 168:439-449

Schnitzler H-U (1968) Die Ultraschall-Ortungslaute der HufeisenFledermäuse (Chiroptera-Rhinolophidae) in verschiedenen Orientierungssituationen. Z Vergl Physiol 57:376-408

Schuller G, Rübsamen R (1981) Laryngeal nerve activity during pulse emission in the CF-FM bat, Rhinolophus ferrumequinum. I. Superior laryngeal nerve (external motor branch). J Comp Physiol 143:317-321

Schuller G, Suga N (1976) Laryngeal mechanisms for the emission of CF-FM-sounds in the Doppler-shift compensating bat, Rhinolophus ferrumequinum. J Comp Physiol 107:253-262

Schuller G, Beuter K, Schnitzler H-U (1974) Response to frequency shifted echoes in the bat Rhinolophus ferrumequinum. J Comp Physiol 89:275-286

Suga N, Schlegel P, Shimozawa T, Simmons J (1973) Orientation sounds evoked from echolocating bats by electrical stimulation of the brain. J Acoust Soc Am 54:793-797

Suthers RA, Fattu JM (1973) Mechanism of sound production by echolocating bats. Am Zool 13:1215-1226

Suthers RA, Thomas SP, Suthers B (1972) Respiration, wing-beat and ultrasonic pulse emission in an echolocating bat. J Exp Biol 56:37-48

Suzuki M, Kirchner JA (1969) The posterior cricoarytenoid as an inspiratory muscle. Ann Otol Rhinol Laryngol 78:849-864

Suzuki M, Kirchner JA, Murakami Y (1970) The cricothyroid as a respiratory muscle. Ann Otol Rhinol Laryngol 79:976-983 